\title{
Estratégias de ensino-aprendizagem de programação introdutória no ensino superior: uma Revisão Sistemática da Literatura
}

\author{
Wallace Duarte de Holanda, UFERSA - wallace.holanda@alunos.ufesa.edu.br \\ Laís de Paiva Freire, UFERSA - lais.freire@alunos.ufersa.edu.br \\ Jarbele Cássia da Silva Coutinho, UFERSA - jarbele.coutinho@ufersa.edu.br
}

\begin{abstract}
Resumo: Os elevados níveis de insucesso nas disciplinas introdutórias de programação vem sendo alvo de inúmeras questões e estudos ao longo dos anos. Muitos pesquisadores vêm buscando encontrar soluções que possam contornar este cenário, propondo metodologias e técnicas. Mediante esta problemática, uma Revisão Sistemática da Literatura foi realizada, visando compreender o panorama atual de pesquisa das publicações científicas no Brasil sobre as estratégias adotadas no ensino de programação introdutória em cursos superiores, entre os anos de 2014 a 2018. Os resultados obtidos mostram que softwares educativos, jogos digitais e metodologias específicas estão sendo desenvolvidas e utilizadas com intuito de amenizar os índices de insucesso nessas disciplinas.
\end{abstract}

Palavras-chaves: Informática na Educação, Programação introdutória, Ensinoaprendizagem, Revisão Sistemática da Literatura.

\section{Strategies in Teaching-learning of Introductory Programming in Higher Education: A Systematic Review of Literature}

\begin{abstract}
The high levels of failure in the introductory programming disciplines have been the subject of numerous questions and studies over years. Many researchers have sought to find solutions that could circumvent this scenario by proposing methodologies and techniques. Through this problem, a Systematic Review of Literature was carried out in order to understand the current research landscape of scientific publications in Brazil on the strategies adopted in the teaching of introductory programming in higher education between the years of 2014 to 2018. The results obtained show that educational software, digital games and specific methodologies are being developed and used with the aim of minimizing failure rates in these disciplines.
\end{abstract}

Keywords: Informatics in Education, Introductory Programming, Teachinglearning, Systematic Literature Review.

\section{Introdução}

Ao ingressar no ensino superior, mais precisamente em cursos da área de computação, os alunos se deparam com disciplinas de programação introdutória, responsáveis por introduzir os conceitos fundamentais para aprendizagem de programação ao longo do curso. De acordo com Gomes (2008), essas disciplinas acabam requisitando certas habilidades dos alunos, tais como: interpretação, raciocínio lógico, abstração e a resolução eficaz de problemas. Tais habilidades são imprescindíveis para a compreensão e uma adequada aplicação dos conteúdos estudados em disciplinas de programação.

Silva e Trentin (2016) enfatizam que, em termos gerais, há uma grande dificuldade dos alunos em compreender e aplicar os conceitos de programação, principalmente na criação de algoritmos que resolvam problemas concretos. 
Alguns estudos [Girrafa e Mora, 2013; Holanda et al., 2018] vêm revelando que as disciplinas de programação introdutória, principalmente, tendem a apresentar um alto índice de retenção, conduzindo os alunos à desmotivação para continuidade na disciplina ou, até mesmo, no referido curso de graduação. Os elevados níveis de insucesso em disciplinas de programação introdutória têm motivado a realização de várias pesquisas ao longo dos anos (Gomes, 2008), resultando em propostas de metodologias, técnicas e ferramentas de suporte ao ensino e aprendizagem de programação.

Diante desse contexto, este trabalho tem como objetivo reunir e analisar as principais estratégias que apoiam o processo de ensino-aprendizagem de programação introdutória em cursos de ensino superior. Assim, definimos a seguinte questão de pesquisa: Qual é o panorama atual de pesquisa das publicações científicas no Brasil, nos últimos 5 anos (2014-2018), que apresentam estratégias para auxiliar o ensinoaprendizagem de programação introdutória em cursos de nível superior?

Deste modo, para alcançar tal objetivo, uma Revisão Sistemática de Literatura (RSL) foi conduzida, através da análise de artigos inerentes ao contexto deste estudo, em cinco importantes eventos nacionais na área de Informática na Educação: a Revista Brasileira de Informática na Educação (RBIE), a Revista Novas Tecnologias na Educação (RENOTE), o Workshop sobre Educação em Computação (WEI), o Workshop de Informática na Escola (WIE) e o Congresso Brasileiro de Informática na Educação (CBIE).

Portanto, as principais contribuições deste artigo consistem na apresentação do panorama atual dos estudos sobre o ensino-aprendizagem de programação introdutória no ensino superior e no compartilhamento dos resultados das experiências realizadas ao longo dos últimos 5 anos, de modo a destacar as principais técnicas, metodologias e ferramentas adotadas.

Assim, este artigo encontra-se organizado da seguinte forma. A Seção 2 discute alguns trabalhos relacionados com a temática deste estudo. A Seção 3 apresenta o protocolo utilizado nesta RSL. Na Seção 4 são apresentados os resultados obtidos. Na Seção 5 os resultados são discutidos. E, na Seção 6 temos as considerações finais.

\section{Trabalhos Relacionados}

Alguns poucos estudos (Silva et al., 2015; Borges et al., 2018) têm usado a RSL com intuito de obter informações sobre metodologias e ferramentas que possam contribuir com a aprendizagem de programação introdutória.

Em Silva et al. (2015) é apresentado uma RSL referente às abordagens para o ensino-aprendizagem de programação, publicados nos anos de 2009 a 2013. Os autores utilizaram eventos/periódicos nacionais como fonte de busca, incluindo, ao final do estudo, 73 trabalhos primários. Os resultados mostraram uma tendência no desenvolvimento de ferramentas para o ensino de algoritmos em cursos de graduação. Dentre as ferramentas listadas, o desenvolvimento de jogos digitais foi o mais destacado.

Em Borges et al. (2018) uma RSL foi conduzida com o intuito de analisar o panorama atual das pesquisas e experimentos relativos às abordagens de ensinoaprendizagem de programação no Brasil, entre os anos de 2012 e 2016. Os autores utilizaram fontes de busca nacionais para a seleção dos trabalhos. Ao todo, 45 artigos foram selecionados. Como resultado, foi verificado que novas ferramentas têm sido propostas e utilizadas em contextos diversificados - no ensino superior, médio e fundamental, por exemplo. Além disto, observou-se a escassez de registros de abordagens do ensino de programação para a Educação a Distância (EaD).

Nossa pesquisa difere-se, das citadas anteriormente, ao selecionar e analisar trabalhos que apresentam técnicas, metodologias e ferramentas relacionadas ao ensino- 
aprendizagem de programação introdutória no contexto do ensino superior, publicados entre os anos de 2014 e 2018. Dessa forma, além de buscar entender o panorama atual da área, uma análise mais específica é realizada com o intuito de identificar estratégias eficientes através dos resultados dos experimentos relatados nos trabalhos, além de apontar possibilidades de estudos a serem realizados.

\section{Método Utilizado}

Segundo Kitchenham (2007), uma RSL é um meio de identificar, avaliar e interpretar todas as evidências científicas disponíveis e relevantes para uma determinada questão de pesquisa, área de tópico ou fenômeno de interesse. O método utilizado para realizar esta RSL foi baseado em um protocolo adaptado do trabalho de Kitchenham (2007).

\subsection{Questões de pesquisa}

A questão central de pesquisa desta RSL consiste em compreender o panorama atual de pesquisa das publicações científicas no Brasil, nos últimos 5 anos (2014-2018), que apresentam estratégias para auxiliar o ensino-aprendizagem de programação introdutória em cursos de nível superior. Para responder essa questão central, foram definidas as seguintes questões de pesquisa específicas $(\mathrm{QE})$ :

- QE1: Quais são as instituições de pesquisa envolvidas na área e como elas estão distribuídas pelo Brasil?

- QE2: Quais as principais técnicas e/ou metodologias utilizadas para auxiliar o ensino-aprendizagem de programação introdutória?

- QE3: Quais ferramentas e/ou softwares educativos têm sido adotados para auxiliar o ensino-aprendizagem de programação introdutória?

- QE4: Quais tipos de estudo têm sido desenvolvidos neste contexto?

- QE5: Quais os principais desafios encontrados nesta área?

\subsection{Processo de busca}

A pesquisa por estudos primários foi realizada através de uma busca manual em cinco fontes de busca da área de Informática na Educação, a citar: RBIE (Revista Brasileira de Informática na Educação), RENOTE (Revista Novas Tecnologias na Educação), WEI (Workshop sobre Educação em Computação), WIE (Workshop de Informática na Escola) e CBIE (Congresso Brasileiro de Informática da Educação). Os critérios para a seleção das fontes de busca foram: (i) disponibilidade de consultar os artigos na web; (ii) importância e relevância das fontes; e (iii) principais conferências e periódicos nacionais na área de Informática na Educação. Não foram adotadas outras fontes de busca, porque o escopo de busca foi limitado às questões de pesquisa (consultar a Subseção 3.1) norteadoras desta RSL.

Para realizar o processo de busca nos anais das fontes de busca, foram selecionados um conjunto de strings de busca baseados em termos que representam o contexto da pesquisa. Assim, foi definida a seguinte string de busca: (ensino OR aprendizagem) AND (programação básica OR programação introdutória) AND (ensino superior).

\subsection{Seleção e avaliação}

O processo de seleção e avaliação dos artigos foi dividido em quatro estágios. A préseleção (denominada estágio 0 ) dos artigos consistiu em verificar os anais das referidas fontes de busca e acessar manualmente todos os artigos completos, através da análise dos seus títulos, resumo e palavras-chave, considerando a string de busca supracitada. Os 
artigos pré-selecionados foram analisados com base nos critérios de inclusão (CI) e nos critérios de exclusão (CE) apresentados no Quadro 1.

Quadro 1. Critérios de Inclusão e Critérios de Exclusão.

\begin{tabular}{|c|c|}
\hline Critérios de Inclusão & Critérios de Exclusão \\
\hline $\begin{array}{l}\text { CI1: Artigos com foco no contexto desta RSL; } \\
\text { CI2: Artigos publicados entre } 2014 \text { e 2018; } \\
\text { CI3: Artigos que abordam, pelo menos, uma } \\
\text { prática didática e/ou propõe alguma } \\
\text { metodologia e/ou alguma ferramenta de } \\
\text { ensino de programação introdutória. }\end{array}$ & $\begin{array}{l}\text { CE1: Artigos repetidos em mais de uma fonte } \\
\text { de busca; } \\
\text { CE2: Artigos sem acesso disponível; } \\
\text { CE3: Artigos incompletos; } \\
\text { CE4: Artigos com conteúdos irrelevantes à } \\
\text { pesquisa; } \\
\text { CE5: Artigos que abordam o processo de } \\
\text { ensino-aprendizagem de programação } \\
\text { introdutória fora do contexto do ensino } \\
\text { superior. }\end{array}$ \\
\hline
\end{tabular}

No estágio 1 foram analisados o título, o resumo e as palavras-chave de acordo com os critérios de exclusão CE1, CE2, CE3, CE4 e CE5. Os trabalhos primários incluídos ou duvidosos foram adicionados à Lista de Candidatos (LC1).

No estágio 2, foi realizada a leitura do título, resumo, palavras-chave, introdução e conclusão dos trabalhos da LC1. Foram considerados os critérios de exclusão CE1, CE3 e CE5. Os estudos rejeitados, de acordo com os critérios de exclusão foram catalogados na Lista de trabalhos Excluídos (LE2), os trabalhos duvidosos foram adicionados à Lista de trabalhos Duvidosos (LD) e os trabalhos incluídos foram inseridos na Lista de trabalhos Candidatos Incluídos (LC2).

O estágio 3 consistiu em catalogar os trabalhos da LC2 de forma a construir uma lista final de trabalhos devidamente classificados de acordo com os CI estabelecidos. Neste estágio, os trabalhos foram analisados a partir da leitura completa do texto, podendo, ainda, serem catalogados como rejeitados ou duvidosos. Os trabalhos incluídos fizeram parte da Lista de trabalhos Incluídos (LI) e compuseram os quantitativos da pesquisa.

\subsection{Avaliação da Qualidade}

A avaliação da qualidade dos trabalhos primários foi realizada a partir de uma pontuação final atribuída para cada artigo, com base em questões de qualidade. A pontuação das questões basearam-se em: $\mathrm{Y}(\operatorname{sim})=1, \mathrm{P}$ (parcialmente) $=0.5$ e $\mathrm{N}$ (não) $=0$, de acordo com Kitchenham (2007). Os artigos com pontuação 0 foram retirados do estudo ${ }^{1}$. Na RSL foram utilizadas quatro questões de avaliação (QA), são elas:

- QA1: O artigo descreve o método de pesquisa empregado no estudo?

- QA2: Foi utilizada alguma ferramenta ou software educativo para auxiliar a abordagem apresentada?

- QA3: O artigo destaca pelo menos uma estratégia, ferramenta ou metodologia para o ensino-aprendizagem de programação introdutória?

- QA4: Os resultados apresentados referem-se a estudos realizados com alunos ingressantes de cursos de Computação e/ou afins?

\footnotetext{
${ }^{1}$ Acesso:https://docs.google.com/document/d/17-

WfcVOu3gc62yHgTnxLgXkeFi1R_lh_fpAycqLck7I/edit?usp=sharing
} 


\section{Resultados Obtidos}

Nesta seção são apresentados os resultados obtidos com esta RSL, de modo a categorizálos em informações gerais extraídas dos trabalhos primários e informações específicas às questões de pesquisa definidas na Seção 3.1.

\subsection{Informações gerais sobre os trabalhos primários}

A busca manual resultou em 187 trabalhos primários coletados no estágio 0,81 selecionados no estágio 1, 45 selecionados no estágio 2, dos quais 51 foram incluídos no estágio 3. A Tabela 1 apresenta o resultado geral da busca realizada, considerando: os trabalhos disponíveis em cada fonte de busca analisada; os trabalhos pré-selecionados no estágio 0 ; e, os artigos incluídos ${ }^{2}$, resultantes do processo de seleção realizado durante os estágios 1,2 e 3 desta RSL.

Tabela 1. Quantidade de artigos por fonte de busca.

\begin{tabular}{|c|c|c|c|c|}
\hline Fonte de Busca & $\begin{array}{c}\text { Artigos } \\
\text { Disponíveis }\end{array}$ & $\begin{array}{c}\text { Total de artigos da } \\
\text { RSL }\end{array}$ & $\begin{array}{c}\text { Artigos } \\
\text { Incluídos }\end{array}$ & $\begin{array}{c}\text { Artigos } \\
\text { Incluídos (\%) }\end{array}$ \\
\hline RBIE & 147 & 13 & 7 & $4,7 \%$ \\
\hline RENOTE & 561 & 30 & 9 & $1,6 \%$ \\
\hline WEI & 209 & 54 & 19 & $9,0 \%$ \\
\hline CBIE & 741 & 52 & 13 & $1,7 \%$ \\
\hline WIE & 451 & 38 & 3 & $0,6 \%$ \\
\hline Total de Artigos & 2109 & 187 & 51 & $2,4 \%$ \\
\hline
\end{tabular}

Como visualizado no Gráfico 1, o ano de 2015 concentrou o maior índice de publicações com 29,4\% de trabalhos publicados (equivalente à 15, dos artigos incluídos). Em 2016, encontramos 25,4\% de trabalhos publicados na área, ou seja, 13 artigos. Em 2017 e 2018, tivemos uma concentração de 15,6\% de publicações (equivalente a 8 publicações), em cada ano. Por fim, em 2014, tivemos o menor índice de publicações, 13,7\% (equivalente a 7) de artigos publicados na área desta RSL.

Gráfico 1. Quantidade de artigos por ano de publicação.

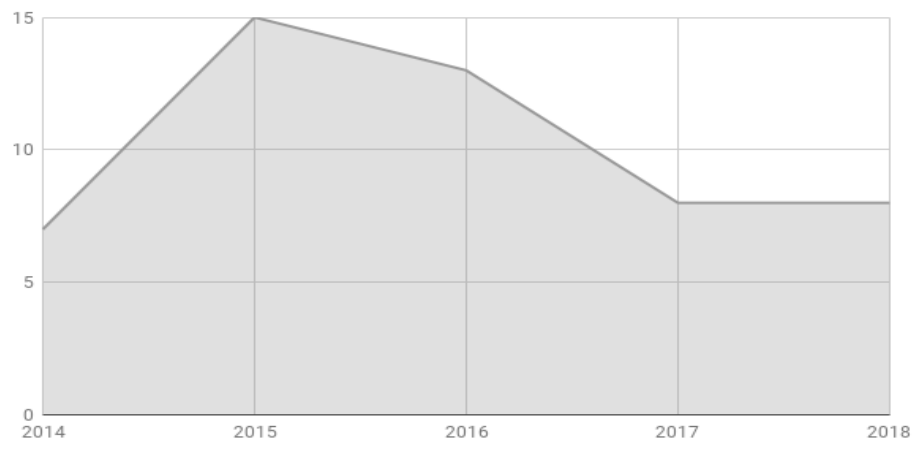

\subsection{Respostas às questões de pesquisa}

Os trabalhos primários resultantes desta RSL foram analisados através da leitura completa do texto e foram extraídas respostas às questões de pesquisa específicas definidas na Seção 3.1 .

\subsubsection{QE1. Quais são as instituições de pesquisa envolvidas na área e como elas estão distribuídas pelo Brasil?}

\footnotetext{
${ }^{2}$ Acesso:https://docs.google.com/document/d/1C1V1YM_bXWcLPOw4Pq1hIsiu8Ls2ihWj2XA0d03K81 E/edit?ts $=5$ cc6 $6 \mathrm{dbcd}$
} 
A revisão encontrou diferentes Instituições de Ensino Superior (IES) que desenvolveram estudos sobre o ensino-aprendizagem de programação introdutória em cursos de graduação. Constatou-se pois, que a região Sul do Brasil é onde se concentra o maior contingente de publicações representando $37 \%$ do total de artigos publicados, sendo o estado do Rio Grande do Sul com o maior número de publicações, um total de dezesseis trabalhos, representando $31 \%$ do total geral de artigos desta RSL, e $84 \%$ do total de publicações da região Sul do Brasil, sendo a Universidade Federal do Pampa (UNIPAMPA) a campeã de publicações, com seis artigos publicados.

$\mathrm{Na}$ região Norte, tem-se trabalhos representantes dos estados do Amazonas com três publicações, totalizando $6 \%$ do total geral, onde destaca-se a Universidade Federal Rural da Amazônia (UFRA), com dois trabalhos; e Maranhão com apenas um artigo, perfazendo $2 \%$ do contingente de publicações. No Nordeste do Brasil somou-se o total de quatorze artigos publicados em sete estados diferentes. Destaca-se, porém, o estado do Rio Grande do Norte com cinco trabalhos, o que representa $36 \%$ das publicações da região e $10 \%$ do total de artigos analisados. Destes, sobressai-se a Universidade Federal do Rio Grande do Norte (UFRN), com quatro publicações, perfazendo $80 \%$ das publicações no estado e $8 \%$ no total de trabalhos analisados nesta RLS.

O Centro-Oeste brasileiro conta com apenas uma publicação, realizada em uma parceria entre o Instituto Federal do Mato Grosso do Sul e a Faculdade Maurício de Nassau do estado da Paraíba, resultando em 2\% das publicações analisadas. Por fim, a região Sudeste conta com um total de treze publicações, o que resulta em $26 \%$ do total geral. Destes, o estado de São Paulo sobressai-se com oito artigos publicados, ou seja, $62 \%$ das publicações da região e $16 \%$ do total de trabalhos analisados, conforme ilustrado no Gráfico 2, abaixo. Informações mais detalhadas podem ser consultadas em: $<$ https://docs.google.com/spreadsheets/d/1BZP1HLw4-

62BovR6p_PPNCwZ3uabHq6iYaNOehIt120/edit?usp=sharing $>$.

Gráfico 2. Quantidade de artigos por região.

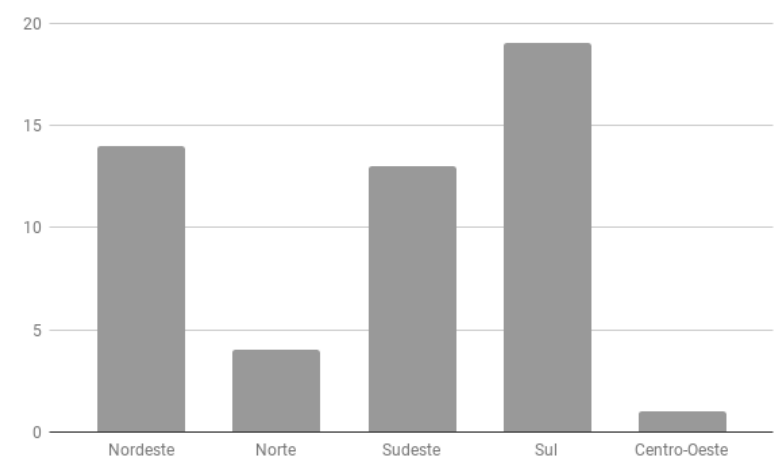

\subsubsection{QE2. Quais as principais técnicas e/ou metodologias utilizadas para auxiliar o ensino-aprendizagem de programação introdutória?}

Dentre as principais metodologias e/ou técnicas apontadas nas pesquisas destacam-se: a utilização de gamificação/ambiente lúdico, o uso de robótica (linguagens específicas e equipamentos), a mediação online, a interdisciplinaridade e abordagens tradicionais de ensino. Outras metodologias foram citadas pelos trabalhos, mas não se enquadram nas categorias supracitadas (Tabela 2). Dentre as metodologias, destacaram-se as estratégias de aprendizagem motivacionais baseadas em robótica (A23, A46, A58, A165 e A195) e baseadas em PBL (A58, A63); bem como, o uso de linguagens de codificação específicas para aprendizagem de lógica de programação, como: Scratch (A43, A68 e A102), 
linguagem Logo (A55) e linguagem PortuCol (A52). Consulte mais detalhes em: $<$ https://docs.google.com/spreadsheets/d/1yDjn4Bm0N10KoULFFior7atZiJeiTt9u2pfyy cLec7o/edit?usp=sharing $>$.

Tabela 2. Descrição das metodologias encontradas.

\begin{tabular}{|c|c|c|c|}
\hline Descrição & Identificadores dos artigos (ID) & Quantidade & Quantidade (\%) \\
\hline $\begin{array}{l}\text { Gamificação/ } \\
\text { lúdico }\end{array}$ & $\begin{array}{c}\text { [A9], [A16], [A21], [A37], [A40], [A54], } \\
\text { [A55], [A63], [A65], [A68], [A73], } \\
\text { [A102], [A107], [A119], [A136], [A170], } \\
\text { [A172], [A183] }\end{array}$ & 18 & $35 \%$ \\
\hline Robótica & [A23], [A46], [A58], [A165], [A195] & 3 & $6 \%$ \\
\hline Mediação online & $\begin{array}{c}\text { [A8], [A10], [A24], [A59], [A67], [A117], } \\
\text { [A198] }\end{array}$ & 7 & $14 \%$ \\
\hline Interdisciplinar & [A43], [A58], [A113], [A123] & 4 & $8 \%$ \\
\hline Tradicional & $\begin{array}{c}{[\mathrm{A} 13],[\mathrm{A} 23],[\mathrm{A} 46],[\mathrm{A} 49],[\mathrm{A} 52],} \\
{[\mathrm{A} 171]}\end{array}$ & 6 & $12 \%$ \\
\hline Outros & $\begin{array}{c}\text { [A2], [A3], [A11], [A20], [A27], [A31], } \\
{[\mathrm{A} 66],[\mathrm{A} 101],[\mathrm{A} 108],[\mathrm{A} 141],[\mathrm{A} 165],} \\
{[\mathrm{A} 179],[\mathrm{A} 195]}\end{array}$ & 13 & $25 \%$ \\
\hline
\end{tabular}

4.3 QE3. Quais ferramentas e/ou softwares educativos têm sido adotados para auxiliar o ensino-aprendizagem de programação introdutória? Dentre os artigos selecionados, foi constatado que 70,5\% (36 artigos) apontaram o uso de alguma ferramenta ou software educativo no apoio o ensino-aprendizagem de programação introdutória. Ambientes de aprendizagem online (A8, A10, A24, A59, A63, A67, A117, A198) destacaram-se como um dos principais meios utilizados nas IES. Jogos digitais, também tem sido uma prática bastante comum nas IES para o ensino de programação introdutória. Destacaram-se os jogos digitais: GameLogic [A37], Defense of the Ancients 2 [A136], EGameFlow [A54] e Teddy Race [A21].

Ferramentas de simulação e correção de código (A16, A176) também foram utilizadas, permitindo aos estudantes, em seus primeiros contatos com a linguagem de programação, maior abstração da complexidade envolvida na correção de erros durante o processo de programação. Os Ambientes de Desenvolvimento Integrado (IDEs) também foram empregados, destacando-se: Portugol Studio [A65] e o Game Maker [A170], ambas contendo configurações adicionais voltadas para auxiliar os alunos iniciantes em suas primeiras experiências com lógica de programação.

Dentre as 36 ferramentas encontradas ${ }^{3}, 11$ (31\%) foram projetadas para uso desktop, $13(36 \%)$ para uso web, 2 (5\%) ferramentas desenvolvidas para a plataforma mobile android e $10(28 \%)$ ferramentas não tiveram a sua plataforma informada. $\mathrm{O}$ Gráfico 3 apresenta essas informações quantificadas.

\footnotetext{
${ }^{3}$ Acesso:https://docs.google.com/spreadsheets/d/1bOPJorG8xBM6XuihrOGMTDMYwTgkxn0JJzatcqIF Q6k/edit?usp=sharing
} 
Gráfico 3. Descrição da disponibilidade das ferramentas encontradas.

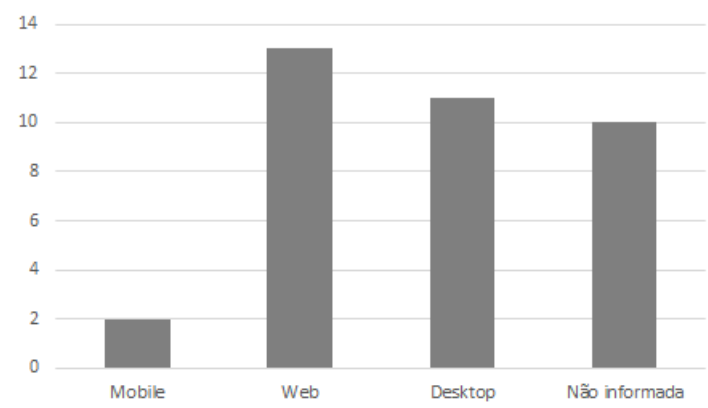

\subsection{QE4. Quais tipos de estudo têm sido desenvolvidos neste contexto?}

Nesta RSL, identificamos e categorizamos 8 tipos de pesquisas desenvolvidas nos artigos, a citar: Revisão Sistemática de Literatura (RSL), Mapeamento Sistemático (MS), Survey, Estudo de Caso, Experimento (pesquisa experimental), Validação de Software, Estudo Qualitativo e Estudo Empírico.

Percebeu-se que as pesquisas mais desenvolvidas no contexto de ensinoaprendizagem de programação introdutória em IES, se deram através da realização de Experimentos (24 artigos, ou seja, 47\% do total), Estudos de caso ( 7 artigos, ou 14\%) e Survey (7 artigos, ou 14\%). Os trabalhos que tratam de RSL, bem como os estudos baseados em MS, Validação de Software e Estudo Qualitativo contam com 2 artigos, cada um $(10 \%)$ do total de artigos, para cada tipo de pesquisa citada. Apenas 1 trabalho referese à realização de um Estudo Empírico, representando 5\% do total de artigos. Por fim, outros quatro trabalhos não informam qual tipo de estudo foi desenvolvido, representando $20 \%$ do total de artigos analisados ${ }^{4}$. A Tabela 3 expõe o tipo de pesquisa e a quantidade de trabalhos destacados em cada caso.

Tabela 3. Descrição da quantidade de estudos encontrados.

\begin{tabular}{|c|c|c|}
\hline Tipo de estudo & Quantidade & Quantidade (\%) \\
\hline Revisão Sistemática & 2 & $3,92 \%$ \\
\hline Mapeamento Sistemático & 2 & $3,92 \%$ \\
\hline Survey & 7 & $13,72 \%$ \\
\hline Estudo de caso & 7 & $13,72 \%$ \\
\hline Experimento (pesquisa experimental) & 24 & $47,04 \%$ \\
\hline Validação de software & 2 & $3,92 \%$ \\
\hline Estudo qualitativo & 2 & $3,92 \%$ \\
\hline Estudo empírico & 1 & $1,96 \%$ \\
\hline Não foi informado & 4 & $7,84 \%$ \\
\hline
\end{tabular}

\subsection{QE5. Quais os principais desafios encontrados nessa área?}

Dentre os trabalhos selecionados nesta RSL, apenas 09 deles destacaram dificuldades ou desafios identificados nesta área de estudo - correspondente a $18 \%$ do total. Em contrapartida, os outros $82 \%$ não informaram dificuldades. Dentre os desafios citados, estão: aulas puramente expositivas e exercícios esparsos; a falta de motivação dos alunos, que, em alguns casos, ocorre devido ao baixo desempenho na disciplina; a ausência de mulheres nos cursos superiores de Computação; métodos de avaliação, onde, em alguns casos, uma única atividade é utilizada para medir o grau de aprendizado dos alunos; dificuldade na aprendizagem, ainda fortemente atrelada à adoção de metodologias de

\footnotetext{
${ }^{4}$ Acesso:https://docs.google.com/spreadsheets/d/1HhpIpUIwYyT-rK_mvU7-wFyBUiHwYKgIqUHxVrFGE0/edit?usp=sharing
} 
ensino obsoletas; e, ferramentas não gratuitas, pois boa parte dos alunos das IES não possuem renda para aquisição de tais ferramentas.

\section{Discussão dos resultados}

Mediante os resultados, percebe-se que a busca por soluções que auxiliem o processo de ensino-aprendizagem de programação introdutória em cursos de ensino superior, vem se caracterizando como temática bastante atual, sendo alvo de discussões e trabalhos de inúmeras universidades nacionais. Diante deste cenário, soluções e aplicações estão sendo criadas, baseando-se, na maioria dos casos, em metodologias lúdicas, que permitam ao aluno um contato diferente dos meios tradicionais de aprendizagem.

Mesmo com o crescente número de usuários que possuem dispositivos mobile, a maioria dos pesquisadores ainda buscam desenvolver soluções, em sua maior parte, voltadas para as plataformas web e desktop. Em linhas gerais, pôde-se verificar que as soluções propostas têm sido implementadas e testadas, e que bons resultados também vêm sendo obtidos. Entretanto, na grande maioria dos casos, as ferramentas não foram continuadas e não se encontram disponíveis para utilização com outros alunos.

\section{Considerações Finais}

Neste trabalho, foram apresentados os resultados de uma RSL, buscando identificar o panorama atual de pesquisa das publicações científicas no Brasil sobre as estratégias adotadas no ensino de programação introdutória em cursos superiores, ao longo dos últimos 5 anos. Os resultados obtidos demonstram que: (i) a alta taxa de reprovação e retenção dos alunos nas disciplinas introdutórias de programação não é uma realidade isolada; (ii) as principais dificuldades enfrentadas pelos alunos diz respeito à lógica de programação e à falta de entendimento da sintaxe da linguagem de programação adotada; (iii) as estratégias investigadas propõem novas abordagens no ensino, visando fornecer um suporte para aprendizagem dos alunos; (iv) as pesquisas na área de estratégias voltadas para o ensino de programação vêm crescendo gradualmente; e, (v) novos métodos de ensino têm sido criados e aplicados em cursos de graduação.

Por fim, os trabalhos analisados podem contribuir diretamente sobre como o ensino de programação vem sendo abordado nas IES, e posteriormente, como o ensino de programação pode ser aperfeiçoado através das estratégias aqui apresentadas. Além de apontar o panorama geral sobre as pesquisas inerentes ao ensino de programação introdutória em cursos superiores, de forma a oferecer suporte ao desenvolvimento de novos estudos na área, bem como proporcionar aos docentes um compilado de informações úteis no que se refere à estratégias de ensino que tiveram sua eficácia validada, possibilitando sua aplicação na realidade local de cada IES.

Como trabalhos futuros, pretende-se expandir esta RSL para outras bibliotecas digitais, além de explorar algumas das metodologias e ferramentas aqui expostas, para validar seu uso em nosso contexto de ensino.

\section{Referências}

BORGES, R.P.; OLIVEIRA, P.R.F.; LIMA, R.G.R.; LIMA, R.W. A Systematic Review of Literature on Methodologies, Practices, and Tools for Programming Teaching. IEEE LATIN AMERICA TRANSACTIONS. v. 16. n. 5. p.1468-1475, 2018.

GIRAFFA, L.M.; MORA, M.C. Evasão na disciplina de algoritmo e programação: um estudo a partir dos fatores intervenientes na perspectiva do aluno. In: III Conferencia sobre el Abandono en la Educación Superior. Anais. Cidade do México: Universidad Nacional Autónoma de México, 2013. 
GOMES, A.J. Dificuldades de aprendizagem de programação de computadores: contributos para a sua compreensão e resolução. Coimbra: Universidade de Coimbra, 2010. 492p. Tese de Doutorado.

HOLANDA, W.D.; COUTINHO, J.C.S.; FONTES, L.M.O. Uma Intervenção Metodológica para Auxiliar a Aprendizagem de Programação Introdutória: um estudo experimental. In: VII Congresso Brasileiro de Informática na Educação. Anais. Fortaleza: Sociedade Brasileira de Computação, 2018.

KITCHENHAM, B.A. Guidelines for performing Systematic Literature Reviews in Software Engineering. Durham: Universidade de Durham, 2007.

MEDEIROS, T.J.; SILVA, T.R.; ARANHA, E.H.S. Ensino de programação utilizando jogos digitais: uma revisão sistemática da literatura. Revista Novas Tecnologias na Educação. v. 11, n. 3, 2013.

SILVA, B.S.; TRENTIN, M.A.S. Dificuldades no Ensino-Aprendizagem de Programação de Computadores: Contribuições para a sua Compreensão e Resolução. In: Simpósio Nacional de Ensino de Ciência e Tecnologia. Anais. Ponta Grossa: Universidade Tecnológica Federal do Paraná, 2016.

SILVA, T. R.; MEDEIROS, T. J.; ARANHA, E. H. S. Jogos Digitais para Ensino e Aprendizagem de Programação: uma Revisão Sistemática da Literatura. In: XXV Simpósio Brasileiro de Informática na Educação. Anais. Dourados: Sociedade Brasileira de Computação, 2014.

SILVA, T. R.; MEDEIROS, T. J.; MEDEIROS, H.; LOPES, R.; ARANHA, E. Ensinoaprendizagem de programação: uma revisão sistemática da literatura. Revista Brasileira de Informática na Educação. v. 23, n. 1, 2015. 